\title{
Foreword
}

\section{E. Standl}

Type 2 diabetes is an increasingly prevalent, chronic disease occurring in about $4 \%$ of the general population and up to 20 to $30 \%$ in high-risk groups in Western countries. Although Type 2 diabetes is well known to contribute to a variety of microvascular complications, it is also associated with a two- to fourfold increase in the risk of cardiovascular disease compared with the non-diabetic population. In fact, cardiovascular disease accounts for $75 \%$ of all mortality in patients with Type 2 diabetes. However, as less than half of the excess risk from cardiovascular disease in Type 2 diabetes can be attributed to classic risk factors such as dyslipidaemia and hypertension, attention has become increasingly focused on the role of hyperglycaemia particularly in the context of insulin resistance.

There is growing evidence showing that both increased postprandial glucose and fasting plasma glucose contribute equally to overall glycaemic control. This is relevant with regard to postprandial glucose, given the numerous studies suggesting that increased postprandial glucose concentrations

Published online: 30 January 2003

(C) Springer-Verlag 2003 have a pivotal role in the development of cardiovascular disease. In particular, epidemiological studies, showing a strong independent relationship between excessive postprandial glucose and an increased risk of cardiovascular disease, have recently gained support from a large meta-analysis involving more than 95000 people from 22 studies. Moreover, increased risk of cardiovascular disease is known to occur early in the disease process and extends throughout the glycaemic spectrum to impaired glucose tolerance and even normal glucose tolerance.

Thus, in order to reduce cardiovascular disease and manage the entire glycaemic risk in Type 2 diabetes, both excessive postprandial glucose and increased fasting plasma glucose concentrations must be controlled, ideally early in the development of the disease. Data clearly show that this can be achieved using agents with complementary modes of action that either restore the early phase of insulin secretion (e.g. nateglinide) or improve insulin sensitivity (e.g. metformin). Successful treatment, and long-term control of diabetes, can only be achieved through a clear understanding of the underlying mechanisms of the disease and are therefore the goals of treatment. Companies such as Novartis have played a key role through their considerable efforts to increase awareness and understanding of postprandial glucose and other factors integral to the control of Type 2 diabetes. 